\title{
Polymeric nanocarrier systems for photodynamic therapy
}

\author{
Li Li and Kang Moo Huh
}

\begin{abstract}
Photodynamic therapy (PDT) is an emerging treatment modality that involves the combined action of photosensitizers (PSs) and light for treatment of solid tumor and other diseases. Although this therapeutic method has been considered as an alternative to classical cancer treatments, clinical PDT requires further advances in selectivity and therapeutic efficacy to overcome numerous shortages related to conventional PDT. In this regard, great efforts have been devoted to the development of polymeric nanocarrier-encapsulated PSs for targeted PDT, aiming at improvement of water solubility and tumor-specificity of hydrophobic PSs. Here, we discuss the general concepts and considerations of polymeric nanocarriers for efficient delivery of PSs. In recent, the amphiphilic PS-polymer conjugate-based self-quenchable nanoparticles and PS-polymer-conjugate/quencher nanocomplexes have emerged as an attractive delivery platform for efficient and reliable PDT. They can incorporate and deliver the PS in a photodynamically inactive state but demonstrate cytotoxic effects by tumor environment-sensitive activation mechanisms, so that the photodynamic cancer treatment can achieve maximum target specificity. Here, we report the recent achievements on the development of activatable PS formulations based on PS-polymer conjugates.
\end{abstract}

Keywords: Photodynamic therapy, Photosensitizer, Drug delivery, Polymer, Nanocarrier, Conjugates

\section{Introduction}

Photodynamic therapy (PDT), a light-activated chemotherapeutic treatment, has emerged as an innovative clinical modality for tumors and nononcological diseases $[1,2]$. This modality is based on the selective retention of a previously administrated photosensitizing molecule in a target site and a measured light dose of appropriate wavelength is then used to irradiate the target tissue. Upon light irradiation, the photosensitizing molecule interacts with molecular oxygen to generate various reactive oxygen species to damage target cells via apoptosis and necrosis $[2,3]$. PDT offers several advantages over conventional therapies for malignant diseases [4-6]. For example, PDT is a minimally invasive method that destroy target cells without surgical risks, serious damages, and systemic complications. Since PSs are typically harmless without light, tumor site treatment can be precisely targeted by selective illumination, thus, PDT is highly selective and targeted in action. PDT can be applied repeatedly without initiating resistance or exceeding total dose limitation (as

\footnotetext{
*Correspondence: khuh@cnu.ac.kr

Department of Polymer Science and Engineering, Chungnam National University, 99 Daehak-ro, Yuseong-gu, Daejeon 305-764, Republic of Korea
}

associated with radiotherapy). Over the past decade, PDT is gradually becoming a more widely used clinical technique and has received regulatory approval for treatment of a number of solid tumors [7], such as lung [8], bladder [9], head and neck [10], ovarian [11], prostate [12], skin [13] and bone carcinomas [14].

The photosensitizing molecules, named as photosensitizers (PSs), function as catalysts when they absorb visible light and convert molecular oxygen to a range of highly reactive oxygen species (ROS) (singlet oxygen and free radicals, such as $\mathrm{OH}^{-}, \mathrm{O}_{2}{ }^{2-}$ and $\mathrm{O}_{2}{ }^{-}$). The detailed mechanism of action of PDT using PS is illustrated as shown in Figure $1[7,15]$. Briefly, PS has a stable electronic configuration with a singlet state in their lowest or ground state energy level $\left(\mathrm{PS}^{0}\right)$. Upon activation, the PS in its ground state absorbs a photon and is promoted into an excited singlet state $\left({ }^{1}{ }^{P S}\right)$. The excited singlet state can relax back to ground state by emitting a fluorescent photon or can convert to the triplet state $\left({ }^{3} \mathrm{PS}\right)$ via intersystem crossing which involves a change in the spin of an electron. This triplet state is a photoactive state, which can interact with molecular oxygen and produce reactive oxygen species by undergoing two main reactions, Type I and Type II 

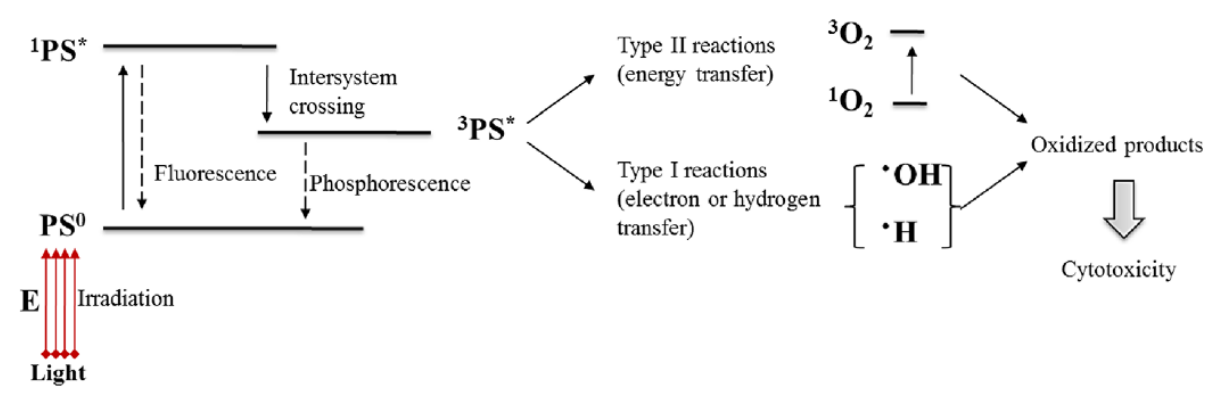

Figure 1 Mechanism of PDT cytotoxicity: photophysical and photochemical reactions represented by modified Jablonski diagram.

reactions. In Type I reaction, the PS transfers an electron to various receptor molecules, producing free radicals or superoxide ions resulting from hydrogen or electron transfer. Type II reaction leads to produce the electronically excited and highly reactive state of oxygen known as singlet oxygen. In PDT, Type II processes are most relevant, and the generation of singlet oxygen is responsible for the irreversible damage of tumor cells [16]. In addition to directly killing tumor cells through production of ROS under light irradiation [17], PDT also can damage the tumor-associated vasculature leading to tumor infarction [18], and can activate the immune response against tumor cells $[19,20]$.

In a PDT process, PSs are critical to the successful eradiation of malignant cells. An ideal PS should meet several crucial requirements [21]: (i) identified purity and composition; (ii) minimal dark toxicity; (iii) photostability; (iv) strong absorbance in a near-IR spectrum range with high extinction molar coefficient; (v) water solubility; (vi) tumor site target specificity; (vii) adequate clearance rate from the body. The first generation PS refers to Hematoporphyrin (Hp) and Photofrin ${ }^{\circ}$ (hematoporphyrin derivative, $\mathrm{HpD}$ ) [22]; and, Photofrin is the first PDT agent approved for clinical use. It has been considered as a therapy against various cancers, such as lung, esophageal bladder, brain, breast, and early-stage cervical cancer. However, although Photofrin has demonstrated significant therapeutic effects, it still suffers from several drawbacks: first, Photofrin is a complex and undefined mixture of dimeric and oligomeric compounds having poor tissue penetration due to its relatively weak absorbance in the red region of the spectrum; second, it has a poor selectivity in terms of target tissue/ healthy tissue ratios; third, it has a low molar extinction coefficient that requires use high doses of Photofrin and light for adequate tumor eradication; forth, it readily accumulate and stay in skin for a longtime, causing longlasting cutaneous photosensitivity [23]. To address these problems, a wide variety of second generation PSs, such as porphyrin derivatives [24,25], phthalocyanines [26-28], and chlorins $[29,30]$, have been developed. Comparing to the first generation, they have the advantage of being pure and well characterized; they can effectively generate singlet oxygen and have absorption maxima at wavelength longer than $630 \mathrm{~nm}$, at which light penetration in tissue is enhanced; their relative high selectivity for malignant sites and fast elimination from the body leads to a reduced skin photosensitivity [22].

Although second generation PSs have addressed several problems with first generation PSs, they still face the challenges associated with the PS delivery [31]. Most existing second generation PSs are aromatic and hydrophobic in nature with poor or limited solubility in water and hence difficult to be intravenously administrated. Even in the case of water-soluble PSs, the poor accumulation selectivity at malignant sites makes them far from clinical applications. Formulating hydrophobic PSs using lipidic (e.g. Cremophor) or organic (e.g. propylene glycol) excipients is reasonable for topical or local administration, but can cause unpredictable biodistribution, toxicity, and hypersensitivity if administrated intravenously [15]. The unpredictable biodistribution can lead to a high plasma retention and unexpected accumulation of PSs in healthy tissues like skin, which often results in accidental damage on blood vessels or non-disease tissues when the optical equipment applied, and/or skin photosensitivity when the patients expose to the sunlight or strong indoor lights. In addition, as for excipients like Cremophor, the issues with allergy, hypersensitivity reactions, and nephrotoxicity have been often reported [32,33]. Therefore, a challenge in PDT for treatment of malignant diseases is to design a safe and tumor-specific carrier platform for systemic delivery of PSs.

To date, a variety of macromolecular nanocarrier platforms such as liposomes [34-36], polymeric nanoparticles [37-41], and micelles [42-45] have been investigated for their potential application in PS delivery (Figure 2), which can provide an effective solution to overcome the shortages of current PSs associated with intravenous administration and selective delivery of the PS to the tumor sites. Beneficial effects of these nanocarriers lie in their excellent colloid dispersity in water that enables solubilization of 

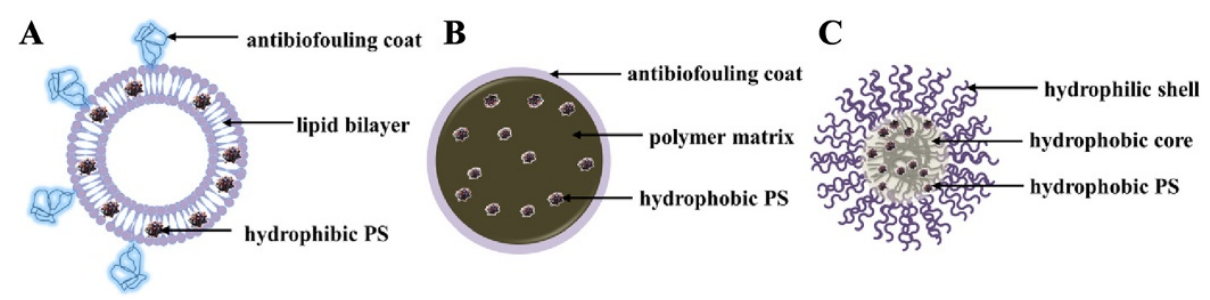

Figure 2 A schematic diagram of PS-encapsulated polymeric nanocarriers: (A) liposome, (B) polymeric nanoparticle, and (C) polymeric micelle.

hydrophobic PS in physiological condition and the enhanced accumulation at tumor sites through enhanced permeability and retention (EPR) effect (Figure 3) [46,47]. Generally, PS can be encapsulated using these carriers by both physical methods using hydrophobic or electrostatic interactions between PS and polymers and chemical methods using various conjugation reactions of PSs to polymers and nanoparticles. The general concepts and considerations of polymeric nanocarriers for delivery of PS will be resumed. In addition to the delivery strategies to modulate the pharmaceutical features and biodistribution of PS, a series of amphiphilic PS-polymer conjugate-based self-quenchable nanoparticles [48-52] and PS-polymerconjugate/quencher nanocomplexes [53-55] have been recently developed. A common characteristic feature of these platforms is that they can incorporate and deliver the PS in a photodynamically inactive state and create active forms and produce cytotoxic effects only at the tumor site. Since the direct tumor cell destruction essentially depends on the in situ generation of cytotoxic singlet oxygen, a controllable singlet oxygen production with high selectivity and localization would lead to more efficient and reliable PDT, thereby, significantly reducing the associated side effects in PDT, such as skin photosensitivity. In a second part, we will describe the strategies that involved in construction of activatable PS formulations based on polymer-PS conjugates and their applications in tumor site-specific PDT.

\section{Review}

\section{Polymeric nanocarriers for delivery of hydrophobic} photosensitizers

To enhance the water solubility and increase the specific accumulation of PSs at the target site, a generally used strategy is encapsulation of the PSs to macromolecular nano-constructs. In this respect, liposomes [34-36], polymeric micelles [42-45], and polymeric nanoparticles [37-41] have been extensively studied for serving as PS carriers in PDT. A common characteristic feature of above mentioned platforms is that they demonstrate

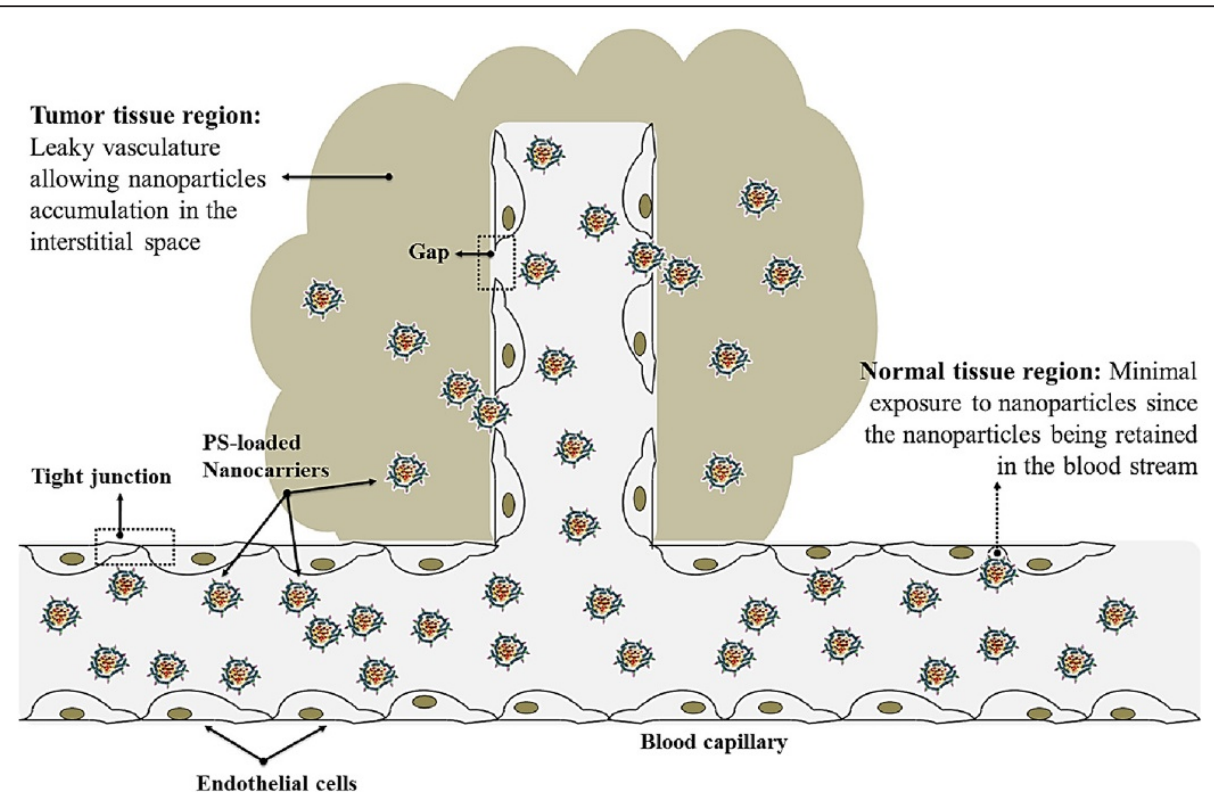

Figure 3 Schematic presentation of passive targeted PDT through EPR effect. PSs encapsulated into nanocarriers can reach tumors selectively through the "leaky" vasculature surrounding the tumors. 
to show tumor-selective accumulation due to the enhanced microvascular permeability and impaired lymphatic drainage in the tumor tissue, a phenomenon which Maeda et al. termed the EPR effect [56,57]. Therefore, systemic PS delivery based on polymeric nanocarrier systems can provide an effective way to not only enhance the water solubility but also modulate the biodistribution profile of hydrophobic PSs and thus facilitating intravenous administration and selective delivery of PS to target tissues.

\section{Liposomes}

Extensive research has been carried out to use liposomes, bilayered phospholipid vesicles, to formulate the hydrophilic or hydrophobic PSs owing to their simple archetypal structures, controllable sizes, and convenient preparation procedure. The hydrophobic PS, such as porphyrin [58] and phthalocyanine derivatives [59], can be dissolved in the phospholipid bilayer region, while the water soluble molecules, such as the prodrug aminolevulinic acid [60], can be encapsulated in the inner core of liposomes. The advantageous of liposome carriers for PS delivery have been described by a number of investigations, which have shown their beneficial effects over other formulations such as simple aqueous dispersions of the PSs. For example, Richter et al. have studied biodistribution and clearance of benzoporphyrin derivative monoacid ring A (BPD-MA) encapsulated into a unilamellar dipalmitoylphosphatidylcholine (DPPC) liposome in comparison to the drug dissolved in a dimethyl sulfoxide solution and a PBS solution [61]. The in vivo study in M1 rhabdomyosarcoma-bearing mice has shown that the maximum concentration of liposome formulation in tumor tissue was obtained $15 \mathrm{~min}$ post-injection against $3 \mathrm{~h}$ for both aqueous formulations. Under light treatment, the liposomal formulation showed significantly more therapeutic efficacy than both aqueous formulations. However, conventional liposomes (unmodified multilamellar or unilamellar liposomes composed of phospholipid) are sometimes suffered from short plasma half-life, which is insufficient time for tumor cell uptake given the rapid elimination by the reticuloendothelial system (RES) and decomposition due to in-body lipid exchange interactions [34]. In this regard, many approaches based on surface modifications of liposomes were explored to produce the long-circulating liposomes featuring substantially enhanced plasma stability. For instance, inclusion of lipids with poly(ethylene glycol) (PEG)-head groups can stabilize the liposome and increase its bioavailability by facilitating evasion of the RES. PEGylated liposomes loaded with chlorin e6 ester have been reported to enhance PDT efficiency compared to the free PS in gastric cancer cell lines in vitro and in vivo [62]. For the long-circulating liposomes, conjugation of the cancer cell targeting moieties, such as ligands, antibodies, proteins and peptides, can effectively increase cancer cell targeting specificity and improve the cellular uptake of liposomes [34,63].

\section{Polymeric nanoparticles}

As an alternative to liposomes, polymeric nanoparticles have been considered as prospective delivery vehicles for PS payloads in PDT. The particle size of polymeric nanoparticles can be easily tailored in the nanometer range by altering the polymer composition and manufacturing processes [64], which is an important factor that enable the PS formulations to be delivered to tumor site through EPR effect and prevent recognition by macrophages and proteins with prolonged circulation time in the blood. In addition, by coating with "stealth" PEG, the nanoparticles can efficiently avoid to be taken up by RES after intravenous administration and consequently increasing the blood circulation time $[65,66]$. Usually, the hydrophobic PSs can be physically entrapped into nanoparticles by hydrophobic or electrostatic interactions between PS and the polymer. Biodegradable polymers, such as polyglycolide (PGA), polylatide (PLA), and their copolymer poly(D,L-lactide-co-glycotide) (PLGA), have been particularly utilized as matrix materials for PS delivery $[40,67]$. The main advantages lie in their versatility, physical robustness, and high drug loading. In addition, their surface properties, morphologies, and composition of polymer matrices can be easily optimized to control the polymer degradation and drug release kinetics for achieving controlled release of PS. As reported by Allemann et al. [68], hexadecafluoro zinc phthalocyanine, was formulated in PEG-coated PLA nanoparticles by the salting out technique with a drug loading of $0.61 \%$. As tested in EMT- 6 tumour-bearing mice at a dose of $5 \mu \mathrm{mol} / \mathrm{kg}$, the PS-loaded nanoparticles caused tumor regression in $100 \%$ of mice compared to only $60 \%$ with the Cremophor EL emulsion. The results indicated that formulation in the biodegradable nanoparticle improved PDT response of the tumor as compared to conventional Cremophor EL emulsion while providing prolonged tumor sensitivity towards PDT. A second generation PS, meso-tetra(hydroxyphenyl) porphyrin (p-THPP) was entrapped into sterile sub-150 nm biodegradable nanoparticles based on three selected polyesters PLGA (50:50 PLGA and 75:25 PLGA) and poly(D,L-lactide) (PLA) using the emulsification-diffusion technique $[69,70]$. The effect of copolymer composition on particle size, drug loading efficiency, and PDT effect has been investigated. A follow-up study was carried out to assess the PDT efficacy of these p-THPP loaded nanoparticles on EMT-6 mouse mammary tumor cells as compared to free p-THPP. The relatively low drug concentration and the short incubation times of nanoparticles with cells required to induce satisfactory photodynamic damages demonstrated that p-THPP loaded 
nanoparticles offer superior photoactivity as compared to the free drug.

\section{Polymeric micelles}

Polymeric micelles are macromolecular complexes formed spontaneously when amphiphilic block or graft copolymers are dispersed in aqueous solution above the critical micelle concentration (CMC). The hydrophobic segments of amphiphilic copolymer exhibit poor compatibility with the aqueous phase and readily assemble to form a core structure for incorporation of hydrophobic drugs. The outer shell region made up by the hydrophilic segments maintains a stabilizing interface between the hydrophobic compartment and the aqueous environment, thus, allowing to solubilize the hydrophobic PSs. In addition, formulating hydrophobic PSs with micelles also could facilitate the control of the drug release in specific site by diffusion, polymer degradation or micelle dissociation mechanisms. Large variety of lipophilic polymers can be used as core forming blocks, such as poly(amino acids) and poly(esters) [71]. However, the corona has almost exclusively been constituted from PEG because of its high water solubility, biocompatibility, and nonfouling properties [72]. Usually, physical incorporation of the hydrophobic PSs can be achieved by various manufacturing processes, such as thin-film hydration, dialysis, or oil-in-water emulsion methods, which lies on the hydrophobic-hydrophobic interaction between drugs and hydrophobic moieties of the copolymer. In PDT, polymeric micelle has been extensively investigated as an alternative platform for delivery of hydrophobic PS and presents several advantages, such as simple preparation, efficient drug loading without chemical modification, and controlled release. Such micelles also exhibit long blood-circulation times and tumor selectivity based on the EPR effect, thus decreasing the unfavorable biodistribution of hydrophobic PSs and consequently reducing the adverse effects like skin photosensitivity. Li et al. reported a formulation of PEGb-poly (caprolactone) (PEG-PCL) micelle incorporated with hydrophobic protoporphyrin IX (PpIX) [73]. The micelles have a high PpIX-loading efficiency of $82.4 \%$ and a narrow size distribution with a mean diameter of $52 \mathrm{~nm}$. In comparison with the free drug, formulation of PpIX in micelles enhanced the total intracellular accumulation of the agent, and thereby markedly increased the photocytotoxicity of PpIX. Hence, the formulation of PpIX in block copolymer micelles may allow the desired PDT efficiency to be achieved at a reduced dose of drug and/or light. The poly(2-ethyl-2-oxazoline)-b-PLA diblock copolymer micelle was used to incorporate meta-tetra(hydroxyphenyl) chlorin (mTHPC) [74]. The m-THPC-loaded micelles exhibited marked PDT effect in vivo, and the therapeutic efficacy was similar to free drug. However, the m-THPC-loaded micelles had less skin phototoxicity after an extended delivery time comparing with free drug. The enhanced tumor specificity of a PS-loaded polymeric micelle could improve therapeutic efficacy while decreasing its adverse effects. Unfortunately, most hydrophobic PSs incorporated into micelles easily form aggregates due to their $\pi-\pi$ interactions and hydrophobic characteristics. Such aggregate formation may severely decrease singlet oxygen generation due to the selfquenching of the excited state. With this regard, incorporation of unaggregated monomeric molecules of PSs into polymeric micelles was considered to retain the therapeutic efficacy of PS's micellar formulation. Knop et al. [75] has reported that the pheophorbide a (PhA)loaded PEG-PCL micelle with $20 \mathrm{~nm}$ in hydrodynamic diameter, corresponding to approximately 200 molecules of polymer and 4 molecules of monomeric $\mathrm{PhA}$ per nano-object, was able to efficiently generate singlet oxygen in the medium. In vitro tests on human cancerous cells have revealed a ca. 2-fold enhanced photocytotoxicity and cellular uptake compared to free $\mathrm{PhA}$.

Indeed, polymeric micelles are attractive carriers for improving PS delivery by enhancing aqueous solubility and retaining the PS payload in the blood for an extended period of time, thus allowing for sufficient EPR-mediated accumulation in the pathological area. However, PSloaded micelles in blood circulation have been found to present a risk of skin photosensitivity, which could lead to damage of endothelial cells or neighboring blood-vessel cells. In order to reduce unwanted photoactivity at non-target sites, we have proposed a new concept for PS-loaded micelles with the ability to prevent singlet oxygen production during the post-treatment period (Figure 4) [76]. As a proof-of-principle of our strategy, PEG-PCL micelles co-loaded with PhA as the singlet oxygen generator and $\beta$-carotene (CAR) as the singlet oxygen scavenger were prepared. We observed that the CAR scavenger in the PhA/CAR micelles significantly diminished PhA-generated singlet oxygen through direct singlet oxygen scavenging in a physiological condition. However, tumor-cell-internalized PhA/CAR micelles exhibited remarkable phototoxicity because of the termination of the scavenging reactions by the intracellular disassembly of the micelle structure and the subsequent drug release. These findings suggest that PhA/CAR micelles could be a promising PDT agent with controllable PDT activity for cancer treatment. Our approach, which co-incorporates a singlet oxygen scavenger in combination with PS into micelles, is an attactive strategy that could permit more accurate PDT to minimize photodamage to non-target tissues, blood cells, or healthy tissues, thus making PDT a safer and more selective clinical technique.

While the polymeric micelle has been extensively investigated as a prospective platform for delivery of 
$\mathbf{A}$<smiles>COCCCCCC(=O)C(C)OCCOC</smiles>

Poly(ethylene glycol)-b-poly(caprolactone) (PEG-b-PCL)

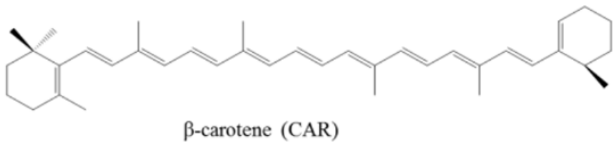

$\beta$-carotene (CAR)

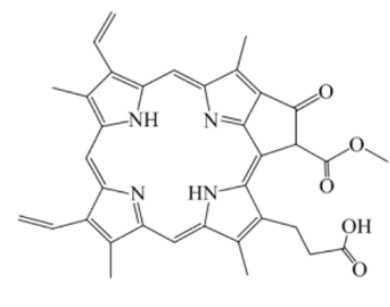

Pheophorbide a $(\mathrm{PhA})$

B

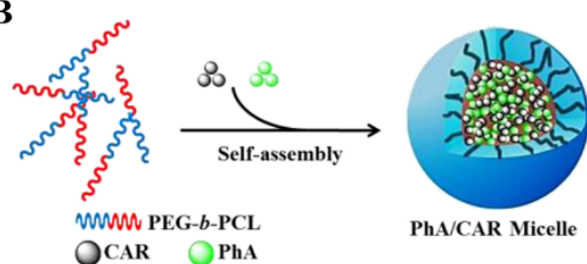

C

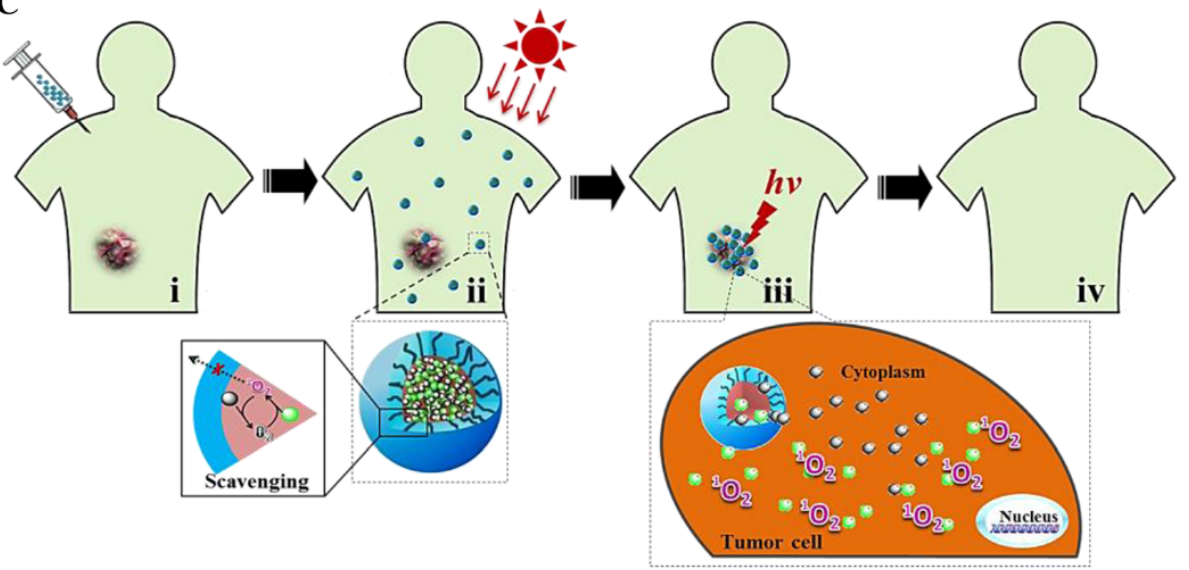

Figure 4 PEG-PCL micelles co-loaded with PhA as the singlet oxygen generator and CAR as the singlet oxygen scavenger for targeted PDT. (A) Chemical structures of PEG-b-PCL copolymer, CAR, and PhA. (B) Co-incorporation of PhA and CAR into PEG-PCL micelle. (C) Schematic presentation of photodynamic cancer therapy using PhA/CAR micelles: i. intravenous administration of drug-loaded micelles; ii. during blood circulation, PhA/CAR micelles is expected to be safe with minimal photodamage to non-target tissues induced by CAR-mediated singlet oxygen scavenging; iii. when the micelles were internalized by cancer cells, PhA and CAR molecules are released from the micelles and then become spatially isolated, resulting in PhA-induced singlet oxygen production and photokilling activity; iv. tumor site restored to health after PDT. Reproduced with permission from: [76].

hydrophobic photosensitizing agent, it has also designed to delivery of a genetically encoded PS for photodynamic cancer treatment [77]. Genetically encoded PS is a recently reported optogenetic tool that enables local ROS production mediated by a fluorescent protein [78]. The unique feature of genetically encoded PS is that cancer cells bear a gene encoding phototoxic protein and produce protein in the adjusted cell compartment, consequently, being expressed in tumor cells rather than administered exogenously [79]. Therefore, the problems of associated with nonspecific PS accumulation in normal tissues could be addressed. Two fluorescent proteins having phototoxic properties are currently known, namely KillerRed (KR) and miniSOG (mini Singlet Oxygen Generator). KR is a dimeric green fluorescence protein (GFP)-like protein that can produce ROS by selective activation using a $585 \mathrm{~nm}$ of light [80]. Another phototoxic protein miniSOG is a small monomeric flavoprotein that can be excited at $448 \mathrm{~nm}$ and produce singlet oxygen with a high yield (0.47) [81]. However, for these fluorescence protein-based PSs, the ROS generation process only affects cells that express proteins, otherwise the cells will remain intact when they are prevented from uptake of the encoding therapeutic plasmids. In this respect, polymeric micelle could be utilized to deliver the protein-based PSs for improving the applicability of genetically encoded PS. In Muthiah's report [77], a quantum dot (QDot)-encapsulated poly(2hydroxyethyl aspartamide) grafted PEG and branched polyethylenimine (PPP) polymeric micelle has been prepared to deliver the KR plasmid to cancer cells. The quantum dot, $\mathrm{CdSe} / \mathrm{ZnS}$, was used as a light harvesting moiety that can facilitate the locally emission of green 
light and subsequently activation of the protein PS within cytoplasm. Intracellular activation of PPP-QDot/KR micelles using a blue light leads to efficient production of ROS; therefore, morphology changes, reduced metabolic activity, and apopotosis of PPP-QDot/KR-treated cancer cells are observed. In addition, by targeting the uptake of PPP-QDot/KR micelles to cancer cells, the cells can be selectively destroyed without affecting normal cells in terms of survival or proliferation. These findings suggest that delivery of the KR using polymeric micelles with multifunctional capacity can be an effective cancer treatment strategy.

\section{Activatable photosensitizer formulations based on polymer-PS conjugates for tumor site-specific PDT}

Based on the mechanism of action of PDT, the direct photodamages on tumor cells highly depend on the in situ generation of cytotoxic singlet oxygen that causes the irreversible damages to pivotal biomacromolecules and other cellular components. Implementation of a controllable singlet oxygen generation with high selectivity and localization would lead to more reliable PDT with minimial phototoxicity to normal cells and hence enhanced efficacy for photodynamic cancer treatment. Above mentioned polymer-based nanocarriers have been considered as targeting platforms for PS delivery. These carrier systems have been demonstrated to show tumor-selective accumulation of PS due to the EPR effect that may improve the therapeutic efficacy of PDT. However, the EPR-based targeting strategies are usually incapable to restrict the localization of PS activation, which may be caused by the unexpected leakage of the PSs during systemic circulation [82]. Therefore, the control of tumor site-specific singlet oxygen production may be essential for avoiding associated side effects of PDT. An activatable PS formulation is a more sophisticated class for PS delivery, which can keep the photoactivity of PS dormant during systemic circulation but demonstrate phototoxicity only at the tumor site [83]. Generally, activatable PSs can be constructed by chemically linking PS with its activation controllers through stimuli-sensitive or cleavable linkers. The linkers between the PS and its activation controller can be specifically degraded by a variety of physicochemical and biological stimuli that exist solely or at very high levels in tumor sites, releasing PS in a photo-active form. PDT with activatable PSs is a promising therapeutic option since it can directly kill cancer cells with reduced side effects in non-target tissues [82-84]. However, current activatable PS formulations are often constructed with a low-molecular-weight system, such as PS-PS [85] or PS-quencher conjugate [86] system, which may suffer rapid elimination from the body and may possess multiple drug resistances, resulting in a short plasma half-life and low bioavailability. In order to address these shortcomings, the activatable formulations based on polymer-PS conjugates have been recently developed. Self-quenchable nanoparticles [48-52] formed by selfaggregation of PS-polymer amphiphilies and PS-polymer conjugate/quencher nanocomplexes [53-55] have been recently reported. These platforms not only possess the common characteristic features of low-molecular-weight activatable systems but also can be maintained stable in the circulatory system for an extended time period due to their appropriate size and surface properties, increasing the possibility for selective accumulation at tumor site through EPR effect.

\section{Self-quenchable nanoparticles}

Some active PDT agents have been based on energy transfer between PSs by control of the aggregation/disaggregation (Figure 5). In this respect, PS-PS self-quenching through fluorescence resonance energy transfer (FRET) mechanism is the deactivation strategy involved in control of the photoactivity of the PSs. FRET is a nonradiative and distance-dependent energy transfer process that based on the interaction between the electronic excited states of two dye molecules in which excitation is transferred from a donor molecule to an acceptor molecule [83]. In recent, a variety of PS-polymer amphiphilies were synthesized for development of self-quenchable nanoparticles (Figure 6) [48-52]. When the hydrophobic PS molecules conjugated to the hydrophilic backbone polymers with an appropriate hydrophilic-hydrophobic balance, the amphiphilic nature of such polymer-PS conjugates could allow them to readily self-assemble to form nano-sized core-shell-structured nanoparticles in aqueous conditions, resulting in $\pi-\pi-$ stacked self-aggregation among hydrophobic PS molecules in the NPs. Under light exposure, PS molecule promotes from its ground state to an excited singlet state, the neighboring PS molecule, as a FRET acceptor, may act on this excited singlet state before the PS enters its triplet state through energy transfer, resulting in the interruption of intersystem crossing. Thus, the pathway for singlet oxygen generation is blocked. As the result of efficient selfquenching, the desired characteristics of self-quenchable nanoparticles can allow the PS molecules to remain photodynamically inactive in the nanoparticulate formulation during systemic circulation to inhibit harmful singlet oxygen generation. In addition, the suppression can be rapidly reversed by molecular stimuli or environment conditions at the tumor site, which allows the PSs to restore the photoactivity and consequently produce cytotoxic species under light exposure.

In recent studies, natural polysaccharides have been popularly used for the development of self-quenchable nanoparticles due to their outstanding merits, such as high water solubility, good biocompatibility, a wide range of molecular weights, and an abundance of sources. 


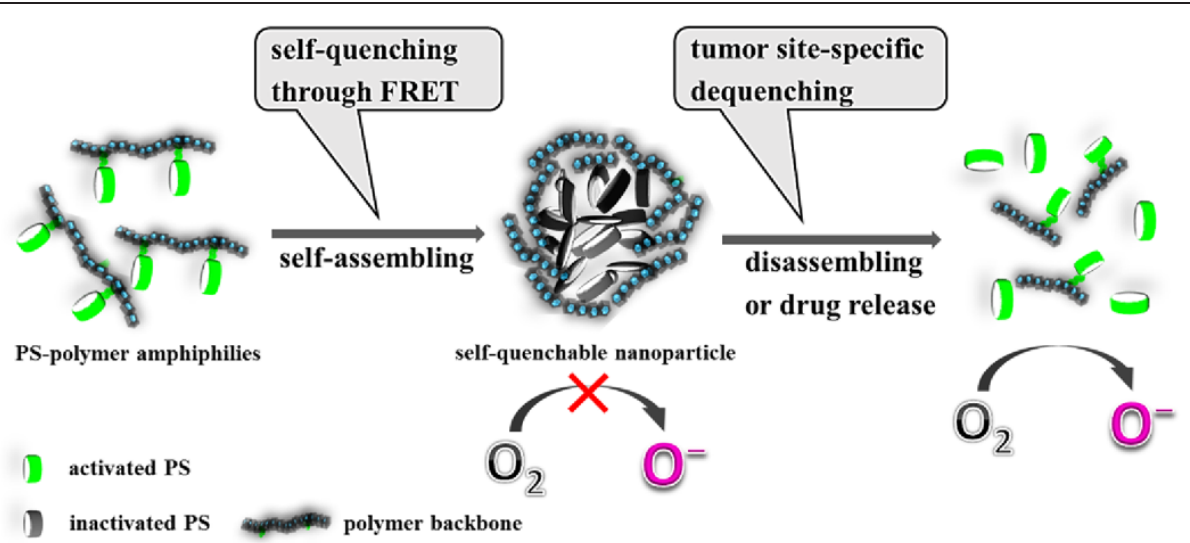

Figure 5 A schematic diagram of self-quenchable nanoparticles. The self-quenchable nanoparticles can incorporate and deliver the PS in a photodynamically inactive state and create active forms and produce cytotoxic effects only at the tumor site.

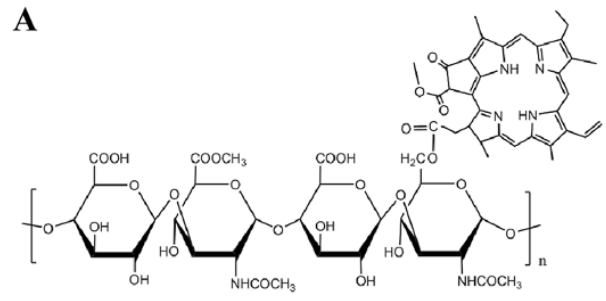

acetylated hyaluronic acid-pheophorbide a conjugate (Ac-HA-PhA)

C

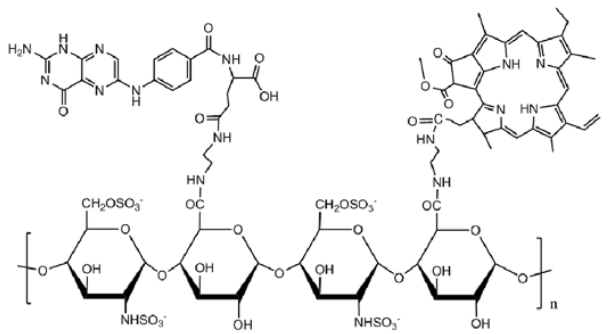

folate-heparin-pheophorbide a conjugate (FHP)

$\mathbf{E}$

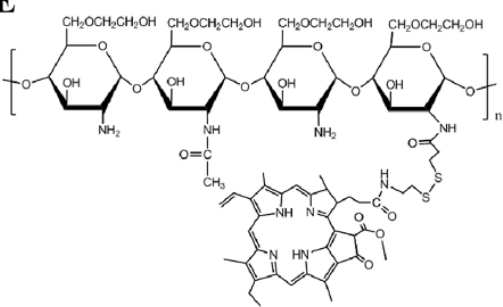

disulfide linked pheophorbide a-glycol chitosan conjugate (PhA-ss-GC)

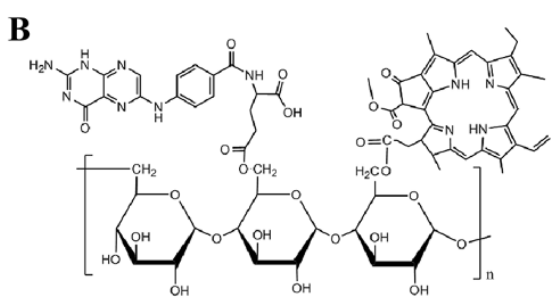

pheophorbide a/folate-pullulan conjugate (PFP)

D

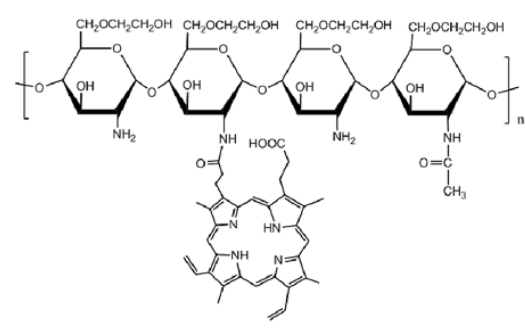

photoporphyrin IX-glycol chitosan conjugate (PpIX-GC)

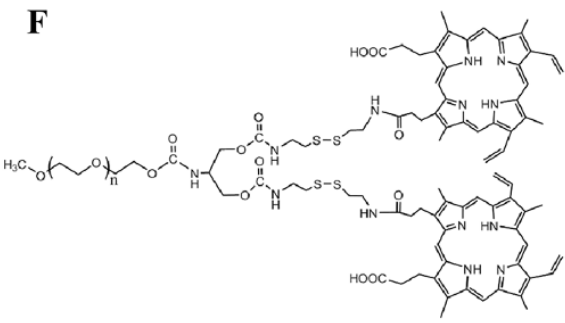

biarmed bioreducible poly(ethylene glycol)-pheophorbide conjugate (PEG-(ss-PhA) $)_{2}$ )

Figure 6 Various of PS-polymer amphiphilies for preparation of self-quenchable nanoparticles: (A) acetylated hyaluronic acid-pheophorbide a conjugate (Ac-HA-PhA) [48], (B) pheophorbide a/folate-pullulan conjugate (PFP) [49], (C) folate-heparin-pheophorbide a conjugate (FHP) [50], (D) photoporphyrin IX-glycol chitosan conjugate (PpIX-GC) [82], (E) disulfide linked pheophorbide a-glycol chitosan conjugate (PhA-ss-GC) [51], (F) biarmed bioreducible poly(ethylene glycol)-pheophorbide conjugate (PEG-(ss-PhA) 2 ) [52]. 
Self-quenchable nanogels synthesized from acetylated hyaluronic acid-PhA (Ac-HA-PhA) conjugates were reported [48]. The Ac-HA-PhA amphiphilic conjugates readily formed $125 \sim 150 \mathrm{~nm}$ nanogels when degree of substitution of PhA molecules per glycosaminoglycan unit of AC-HA varied from 0.31 to 0.08 . The resulting nanogels quenched both the PhA's fluorescence and singlet oxygen generation through a hydrophobic interaction between $\mathrm{PhA}$ themselves. In tumor cells, the photoactivity of Ac-HA-PhA nanogel could be restored, due to the dequenching behavior induced by the enzymatic degradation of polysaccharide backbone, thereby producing singlet oxygen to kill tumor cells under light exposure. Another self-quenchable system based on pullulan/folate-PhA (PFP) conjugates was reported by the same group [49]. The enzyme-treated PFP selfquenchable nanogel showed fluorescence and singlet oxygen generation, which could not be observed in PBS due to the self-quenching effect between PS moieties. In the cell culture system, a dramatic increase in photoactivity induced by disintegration via lysosomal enzymes was observed. In addition, the PFP nanogel exhibited significant phototoxicity to tumor cells, while its cytotoxicity in darkness was negligible in the test range. After subcutaneously injection of PFP nanogels into the non-tumor-bearing Balb/C-nude mice, fluorescence did not immediately appear but was detected after $30 \mathrm{~min}$ and significantly increased for $12 \mathrm{~h}$ due to the dissociation and degradation-induced dequenching of the nanogels. Our group reported synthesis of heparin-PhA (HP) and folateheparin-PhA (FHP) conjugates for preparation of selfquenchable nanoparticles for controllable PDT [50]. HP and FHP conjugates could self-assemble in aqueous media to form nano-sized particles with $130-170 \mathrm{~nm}$ in size. They displayed a self-quenching effect in PBS, while the generation of singlet oxygen dramatically increased in DMF where they exist as dissociated forms. In tumor cells, HP and FHP nanoparticles exhibited marked phototoxicity and were minimally dark-toxic without light treatment. Lee et al. has developed the protoporphyrin IX (PpIX) conjugated glycol chitosan (GC) nanoparticles as a selfquenchable system for PDT [87]. PpIX-GC nanoparticles showed the self-quenching effect with no fluorescence signal and phototoxicity under light exposure, which is due to the compactly crystallized PpIX molecules in the nanoparticles. The switchable photoacitivity of PpIXGC nanoparticles attributes to dequenchability induced by disruption of the condensed nanoparticle structure at the harsh intracellular condition. After intravenous injection into HT-29 human colon adenocarcinoma tumor-bearing mice, they were observed to rapidly and significantly accumulate into the tumor tissue, and displayed strong fluorescence signals and significant therapeutic efficacy according to the effective in-body dequenching properties. By using GC as backbone polymer, we have reported cancer-cell specific PS nano-carrier by synthesizing PhA conjugated GC with reducible disulfide bonds (PhA-ss-GC) [51]. In order to enable quicker release kinetics and a more efficient activation mechanism of the PSs in the tumor cells, such a bioreducible nano-carrier system was especially interested, which might maximize the cytosolic dose of active PSs to achieve higher cytotoxicity, thereby enhancing the treatment efficacy. The relative fast intracellular degradation rate of PhA-ss-GC nanoparticles depends on that the thiol responsive disulfide linkers in the nanoparticles should be rapidly cleaved in the cytosol, as the concentrations of glutathione (GSH) in the cytosol (approximately 2$10 \mathrm{mM}$ ) are much higher than those in extracellular fluids (approximately 2-20 $\mu \mathrm{M}$ ) [88]. The photoactivity of PhA-ss-GC nanoparticles in an aqueous environment was greatly suppressed by the self-quenching effect, which enabled the nanoparticles to remain photoinactive and in a quenched state. However, after the cancer cell internalization, PhA-ss-GC nanoparticles rapidly restored their photoactivity, which is the result of the dissociation of the nanoparticular structure induced by reductive cleavage of the disulfide linkers. Compared to non-reducible PhA-GC nanoparticles with stable amide linkages, PhA-ss-GC nanoparticles presenting higher cytotoxicity with light treatment. In addition, the PhAss-GC demonstrated tumor specific targeting behavior through the EPR effect and enhanced tumor therapeutic efficacy compared to free PhA in tumor-bearing mice.

The above mentioned self-quenchable photosensitizing formulations basically have made up using natural polysaccharides as backbone polymers, but these matrix-forming materials have not been presently approved by Food and Drug Administration (FDA) as safe excipients for anticancer drug delivery, therefore, the development of clinically relevant formulations for controllable PDT is still a challenge. PEG is a nontoxic, nonimmunogenic, nonantigenic, and water-soluble polymer that is approved by the FDA as a safe excipient for ststemic delivery [65]. Utilizing PEG as a backbone polymer may promote the strategy regarding self-quenchable PS carrier system more close towards practical clinical application. In recent, we developed a bioreducible biarmed mPEG-(ss-PhA) $)_{2}$ conjugate for cancer-cell specific photodynamic therapy [52]. PhA molecules were chemically conjugated with biarmed linkages at one end of the MPEG molecule via disulfide bonds. As expected, the amphiphilic mPEG-(ss-PhA) conjugates readily self-assembled to form core/shell nanoparticles in the aqueous media. We observed that the photoactivity of mPEG-(ss-PhA $)_{2}$ nanoparticles was significantly suppressed in the physiological conditions because of their self-quenching properties. However, when the NPs are internalized by cancer cells, the intracellular 
reduction-triggered cleavage of the disulfide bonds accelerates the dissociation of the nanoparticle structure and causes the PhA molecules to restore their photoactivity. The bioreducible activation mechanism of mPEG-(ss$\mathrm{PhA})_{2}$ NPs in cancer cells can efficiently maximize the cytosolic dose of active PSs to achieve high cytotoxicity under light exposure, thereby enhancing the treatment efficacy of photodynamic cancer treatment with reduced side effects.

\section{Polymer-PS conjugate/quencher nanocomplexes}

Besides the self-quenching approach for control of the photoactivity of PSs, the specific deactivation of polymermodified PSs can be achieved by PS-quencher complexation strategy. Generally, this strategy involves in synthesis of water-soluble PS-polymer conjugates, followed by binding to quencher molecules that act as FRET acceptors through covalent or physical bond. A polymeric PS with controllable photoactivity was prepared from a polyelectrolyte complex between cationic PEG-polyethyleniminechlorine e6 conjugate (PEG-PEI-Ce6) and anionic Black Hole Quencher-3 chondroitin sulfate conjugate (BHQ-3CS) (Figure 7) [53]. BHQ-3 was used as dark-quenching material that is able to deactivate the photoactivity of PS by FRET-based photoquenching when it was close neighbored with Ce6 in the nanoparticles. The nanoparticles clearly lost photoactivity by the intermolecular FRET effect in the aqueous phase. However, the quenched photoactivity was restored by the enzymatic degradation of BHQ-3-CS after esterase treatment. In the cell culture system, the rapid cellular internalization and the significant phototoxicity were observed. Recently, we have reported a new GSH-responsive hybrid nanoparticle, PhA-conjugated heparin/gold nanoparticle (PhA-H/AuNP), for controllable PDT (Figure 8) [54]. A thiolated water soluble polymeric PS, PhA-heparin, was

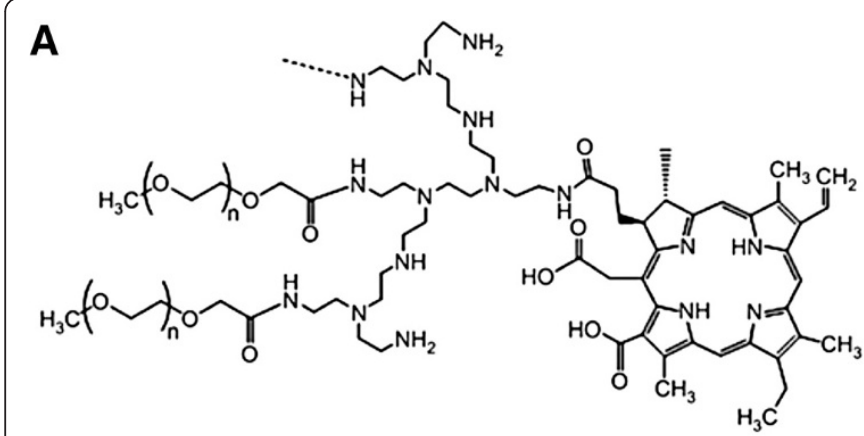

poly(ethylene glycol)-polyethylenimine-chlorine e6 conjugate (PEG-PEI-Ce6)
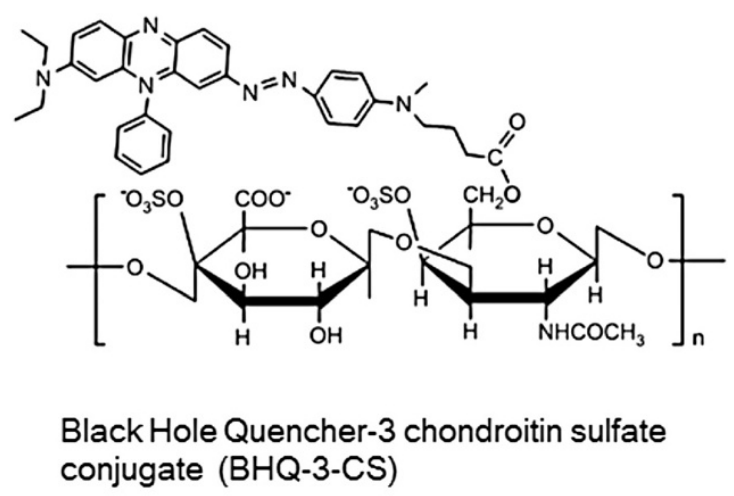

B

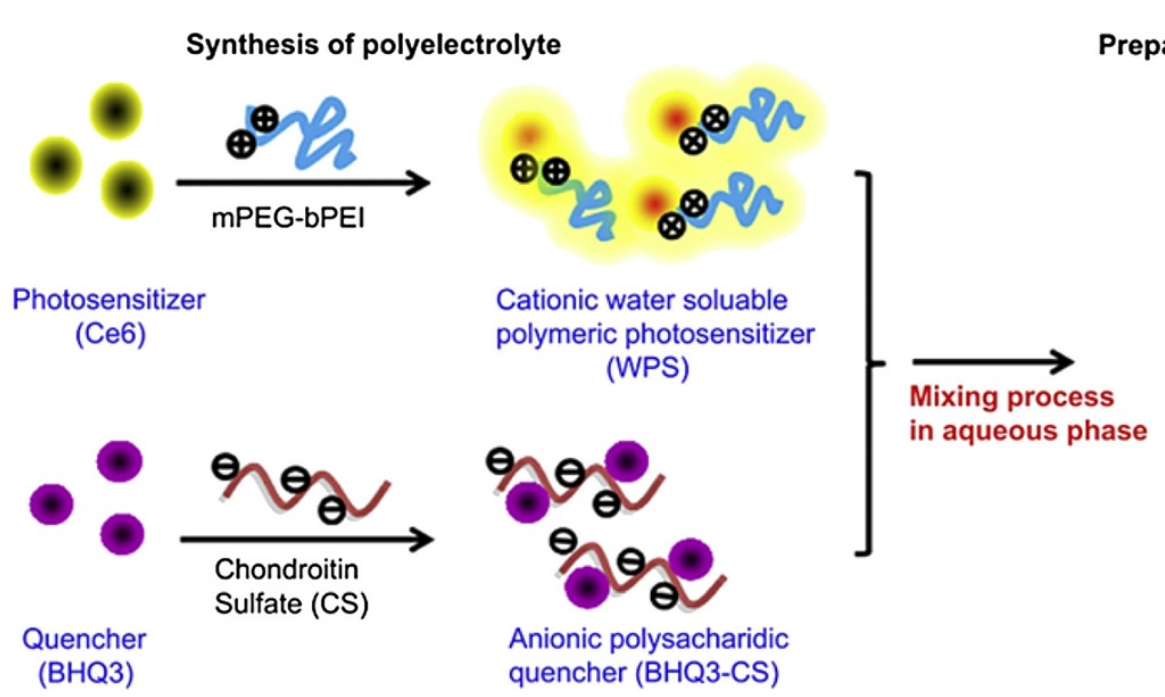

Preparation of NPS

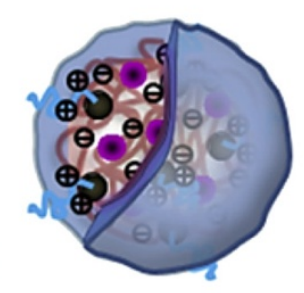

Nano-photosensitizer (NPS)

Figure 7 Polyelectrolyte nanocomplex with controllable photoactivity prepared by ionic interactions between a cationic polymeric PS and an anionic polysaccharide quencher for PDT. (A) Chemical structure of cationic water soluble polymeric PS (poly(ethylene glycol)-polyethyleniminechlorine e6 conjugate (PEG-PEI-Ce6)) and anionic polysaccharide quencher (Black Hole Quencher-3 chondroitin sulfate conjugate (BHQ-3-CS)).

(B) Synthesis of opposite charged polyelectrolyte and facile preparation of nano-PS in the aqueous phase. Reproduced with permission from: [53]. 

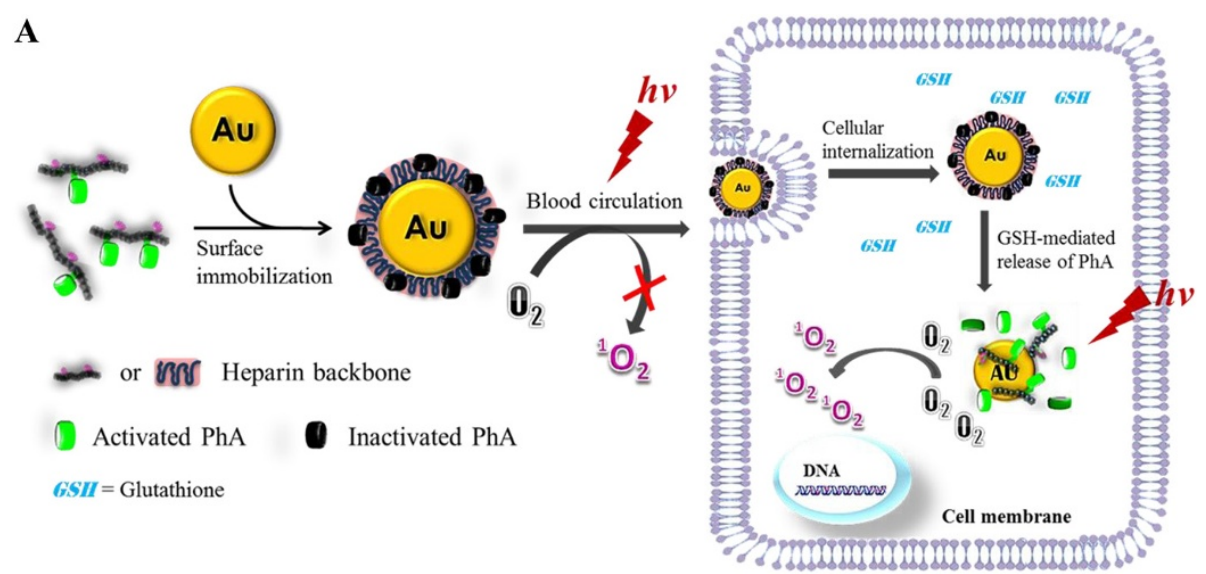

B

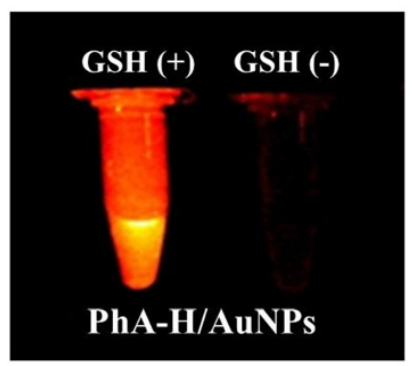

C

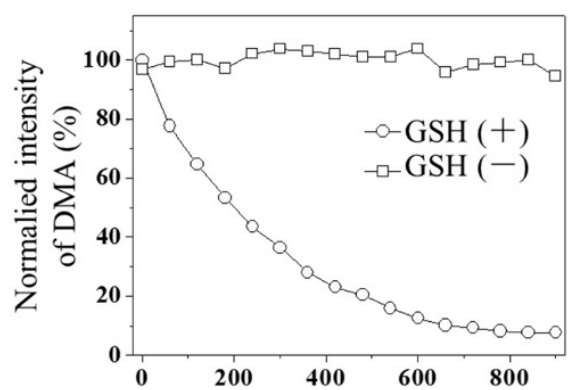

Figure 8 Hybrid PhA-conjugated heparin/AuNP for cancer cell-specific PDT. (A) Schematic diagram of formation of hybrid pheophorbide a/heparin-gold nanoparticle (PhA-H/AuNP) and GSH-mediated photoactivity: PhA becomes photoinactive when immobilized onto the AuNP surface. When PhA-H/AuNPs are internalized by cancer cells, GSH can trigger the release of PhA-H from AuNPs by cleavage of the gold-thiol linkages, causing the recovery of photoactivity of PS payloads. The activated PhA molecules can efficiently generate singlet oxygen to cause cancer cell destruction. (B) And (C), GSH-mediated generation of fluorescence and singlet oxygen, respectively. Reproduced with permission from: [54].

synthesized and immobilized to the surface of AuNP via gold-thiol interaction. AuNP is a well-known quencher for efficient energy transfer based on FRET mechanism for excited dye molecules, which can quench the excited energy of fluorochromes even at a distance of around $40 \mathrm{~nm}$. In our study, AuNP was used not only as a nanocarrier for loading the polymeric PS but also as a quencher material for deactivation of the surface attached PS. Furthermore, the release of PS payloads from the surface of AuNP triggered by GSH-mediated ligand exchange reaction can be expected to cause the effective dequenching processes and thus recovery of the photoactivity. As the result of quenching and dequenching behaviors of PhA-H/AuNP, we observed that the photoactivity was significantly suppressed in aqueous media, but instantaneously restored at the GSH-rich intracellular environment to generate a strong fluorescence signal together with active production of singlet oxygen species with light treatment. In vitro cell tests revealed marked phototoxicity and high intracellular uptake of PhA-H/AuNPs in contrast with free PhA. The PhA-H/AuNPs also exhibited a prolonged circulation characteristic, enhanced tumor specificity, and improved photodynamic therapeutic efficacy compared with free PhA in A549 tumor-bearing mice. As the follow up study, we further prepared a multifunctional hybrid nanoparticle system with a core/shell-structured magnetic $\mathrm{Fe}_{3} \mathrm{O}_{4} / \mathrm{Au}$ nanoparticle and a PhA-heparin surface layer as an activatable platform for PDT [55]. The novelty of the $\mathrm{PhA}-\mathrm{H} / \mathrm{Fe}_{3} \mathrm{O}_{4} / \mathrm{Au}$ hybrid nanoparticle lies in the fact of that unique and inherent surface property of gold shell: (i) suppression of photoactivity caused by the quenchability of the gold shell and (ii) restoration of photoactivity caused by GSH-mediated dequenchability of the surface-conjugated PS molecules. In addition, in vitro MRI studies reveal that the $\mathrm{PhA}-\mathrm{H} / \mathrm{Fe}_{3} \mathrm{O}_{4} / \mathrm{Au}$ nanoparticles could potentially serve as MRI contrast agents in cancer diagnosis and may be used to monitor the photodynamic treatment response used to accurately guide light irradiation.

In a summary, precise control of intracellular site of singlet oxygen production may be essential for efficient tumor cell destruction and reduce the side effect of PDT. Polymer-based activitable PSs are attractive formulations that may enhance therapeutic effect with reduced side effects. They have several advantages for efficient PS delivery: (1) the solubilization of hydrophobic PSs, (2) 
systemic delivery in a photoinactive form, (3) EPR-based tumor selectivity, (4) tumor site-specific generation of cytotoxic species under light exposure.

\section{Conclusion}

PDT has emerged as one of the important therapeutic options in management of cancer. However, there are several shortcomings in the application of conventional PS-based PDT to the treatment of solid tumors. Most clinical applied PSs have limitations such as poor water solubility and low selectivity between tumor and normal tissues for clinical use. Physical encapsulation of PSs into polymer nanocarriers could overcome or reduce inherent limitations that the conventional PS formulations have. PS-encapsulated polymeric nanocarriers may act as a stable formulation for hydrophobic PS under physiological conditions and have an appropriate size for prolonged blood circulation time to deliver PSs to target sites. Moreover, self-quenchable nanoparticles formed by selfaggregation of PS-polymer amphiphilies and PS-polymer conjugate/quencher nanocomplexes have been proposed as a more sophisticated strategy to modulate the photoactivity PS. These PS-polymer conjugates-based formulations can not only engage in solubilization and selective accumulation but also can precisely control the photodynamical reactions only occurring at tumor site, thereby, maximizing the therapeutic efficacy and reducing the side effects related to classic PDT. Although the most of polymeric PS formulations are still undergoing testing in experimental animal models or pre-clinical trials, the above mentioned methodologies for PS delivery hold great potential to bring PDT to the forefront of oncological diseases treatment.

\section{Competing interests}

The authors declare that they have no competing interests.

\section{Authors' contributions}

LL has made substantial contributions to the writing and editing all sections of the manuscript. KMH has contributed significantly by reviewing and suggesting changes overall the article, and has finally checked the manuscript for grammatical and spelling mistakes. Both authors read and approved the final manuscript.

\section{Acknowledgments}

This work was supported by the Basic Science Research Program through the National Research Foundation of Korea (NRF) funded by the Ministry of Education, Science and Technology (2012R1A1A2005029 and 2013R1A2A2A04015914).

Received: 7 August 2014 Accepted: 2 October 2014 Published: 8 December 2014

\section{References}

1. Hamblin MR, Mróz P: Advances in Photodynamic Therapy: Basic, Translational, and Clinical. Boston, Mass: Artech House; 2008.

2. Juarranz A, Jaen P, Sanz-Rodriguez F, Cuevas J, Gonzalez S: Photodynamic therapy of cancer. Basic principles and applications. Clin Trans/ Oncol Off Publ Federation Spanish Oncol Soc National Cancer Institute Mexico 2008, $10: 148-154$
3. MacDonald IJ, Dougherty TJ: Basic principles of photodynamic therapy. J Porphyr Phthalocya 2001, 5:105-129.

4. Dougherty TJ, Gomer CJ, Henderson BW, Jori G, Kessel D, Korbelik M, Moan J, Peng Q: Photodynamic therapy. J Natl Cancer Inst 1998, 90:889-905.

5. Zimcik P, Miletin M: Photodynamic therapy as a new prospective method for cancer treatment. I. History, basic principles. Ceska Slovenska Farmacie Casopis Ceske Farmaceuticke Spolecnosti Slovenske Farmaceuticke Spolecnosti 2004, 53:219-224.

6. Wilson BC: Photodynamic therapy for cancer: principles. Can J Gastroenterol J Can Gastroenterol 2002, 16:393-396.

7. Dolmans DE, Fukumura D, Jain RK: Photodynamic therapy for cancer. Nat Rev Cancer 2003, 3:380-387.

8. Sutedja TG, Postmus PE: Photodynamic therapy in lung cancer. A review. J Photochem Photobiol B 1996, 36:199-204.

9. Senior K: Photodynamic therapy for bladder cancer. Lancet oncol 2005, 6:546.

10. Biel MA: Photodynamic therapy of head and neck cancers. Methods Mol Biol 2010, 635:281-293.

11. Goff BA, Blake J, Bamberg MP, Hasan T: Treatment of ovarian cancer with photodynamic therapy and immunoconjugates in a murine ovarian cancer model. Br J Cancer 1996, 74:1194-1198.

12. Muschter R: Photodynamic therapy: a new approach to prostate cancer. Current Urol Reports 2003, 4:221-228.

13. Roberts DJ, Cairnduff F: Photodynamic therapy of primary skin cancer: a review. Br J Plast Surg 1995, 48:360-370.

14. Guleng GE, Helsing P: Photodynamic therapy for basal cell carcinomas in organ-transplant recipients. Clin Exp Dermatol 2012, 37:367-369.

15. Konan YN, Gurny R, Allemann E: State of the art in the delivery of photosensitizers for photodynamic therapy. J Photoch Photobio B 2002, 66:89-106.

16. Nowis D, Makowski M, Stoklosa T, Legat M, Issat T, Golab J: Direct tumor damage mechanisms of photodynamic therapy. Acta Biochim Pol 2005, 52:339-352.

17. Milla Sanabria L, Rodriguez ME, Cogno IS, Rumie Vittar NB, Pansa MF, Lamberti MJ, Rivarola VA: Direct and indirect photodynamic therapy effects on the cellular and molecular components of the tumor microenvironment. Biochim Biophys Acta 1835, 2013:36-45.

18. Krammer B: Vascular effects of photodynamic therapy. Anticancer Res 2001, 21:4271-4277.

19. Vancikova Z: Principles of the photodynamic therapy and its impact on the immune system. Sb Lek 1998, 99:1-11.

20. Nowis D, Stoklosa T, Legat M, Issat T, Jakobisiak M, Golab J: The influence of photodynamic therapy on the immune response. Photodiagnosis Photodyn Ther 2005, 2:283-298.

21. Detty MR, Gibson SL, Wagner SJ: Current clinical and preclinical photosensitizers for use in photodynamic therapy. J Med Chem 2004, 47:3897-3915

22. O'Connor AE, Gallagher WM, Byrne AT: Porphyrin and nonporphyrin photosensitizers in oncology: preclinical and clinical advances in photodynamic therapy. Photochem Photobiol 2009, 85:1053-1074.

23. Ethirajan $M$, Chen $Y$, Joshi $P$, Pandey RK: The role of porphyrin chemistry in tumor imaging and photodynamic therapy. Chem Soc Rev 2011, 40:340-362.

24. Daicoviciu D, Filip A, Ion RM, Clichici S, Decea N, Muresan A: Oxidative photodamage induced by photodynamic therapy with methoxyphenyl porphyrin derivatives in tumour-bearing rats. Folia Biol 2011, 57:12-19.

25. Pandey RK, Bellnier DA, Smith KM, Dougherty TJ: Chlorin and porphyrin derivatives as potential photosensitizers in photodynamic therapy. Photochem Photobiol 1991, 53:65-72.

26. Spikes JD: Phthalocyanines as photosensitizers in biological systems and for the photodynamic therapy of tumors. Photochem Photobiol 1986, 43:691-699.

27. Moeno S, Krause RW, Ermilov EA, Kuzyniak W, Hopfner M: Synthesis and characterization of novel zinc phthalocyanines as potential photosensitizers for photodynamic therapy of cancers. Photochem Photobiol Sci 2014, 13:963-970.

28. Durmus $\mathrm{M}$, Ahsen $\mathrm{V}$ : Water-soluble cationic gallium(III) and indium(III) phthalocyanines for photodynamic therapy. J Inorg Biochem 2010, 104:297-309.

29. Kreimer-Birnbaum M: Modified porphyrins, chlorins, phthalocyanines, and purpurins: second-generation photosensitizers for photodynamic therapy. Semin Hematol 1989, 26:157-173. 
30. O'Neal WG, Roberts WP, Ghosh I, Wang H, Jacobi PA: Studies in chlorin chemistry. 3. A practical synthesis of $c$, d-ring symmetric chlorins of potential utility in photodynamic therapy. J Org Chem 2006 71:3472-3480.

31. Vrouenraets MB, Visser GW, Snow GB, van Dongen GA: Basic principles, applications in oncology and improved selectivity of photodynamic therapy. Anticancer Res 2003, 23:505-522.

32. Liebmann J, Cook JA, Mitchell JB: Cremophor EL, solvent for paclitaxel, and toxicity. Lancet 1993, 342:1428.

33. Gelderblom H, Verweij J, Nooter K, Sparreboom A: Cremophor EL: the drawbacks and advantages of vehicle selection for drug formulation. Eur J Cancer 2001, 37:1590-1598.

34. Derycke AS, de Witte PA: Liposomes for photodynamic therapy. Adv Drug Deliv Rev 2004, 56:17-30

35. Broekgaarden M, de Kroon Al, Gulik TM, Heger M: Development and in vitro proof-of-concept of interstitially targeted zinc- phthalocyanine liposomes for photodynamic therapy. Curr Med Chem 2013, 21:377-391.

36. Bovis MJ, Woodhams JH, Loizidou M, Scheglmann D, Bown SG, Macrobert AJ: Improved in vivo delivery of $\mathrm{m}$-THPC via pegylated liposomes for use in photodynamic therapy. J Control Release 2012, 157:196-205.

37. Ricci-Junior E, Marchetti JM: Preparation, characterization, photocytotoxicity assay of PLGA nanoparticles containing zinc (II) phthalocyanine for photodynamic therapy use. J Microencapsul 2006, 23:523-538.

38. Ricci-Junior E, Marchetti JM: Zinc(II) phthalocyanine loaded PLGA nanoparticles for photodynamic therapy use. Int J Pharm 2006, 310:187-195.

39. Chatterjee DK, Fong LS, Zhang Y: Nanoparticles in photodynamic therapy: an emerging paradigm. Adv Drug Deliv Rev 2008, 60:1627-1637.

40. Lee YE, Kopelman R: Polymeric nanoparticles for photodynamic therapy. Methods Mol Biol 2011, 726:151-178.

41. Chung CW, Chung KD, Jeong Yl, Kang DH: 5-aminolevulinic acid-incorporated nanoparticles of methoxy poly(ethylene glycol)-chitosan copolymer for photodynamic therapy. Int J Nanomedicine 2013, 8:809-819.

42. Taillefer J, Brasseur N, van Lier JE, Lenaerts V, Le Garrec D, Leroux JC: In-vitro and in-vivo evaluation of $\mathrm{pH}$-responsive polymeric micelles in a photodynamic cancer therapy model. J Pharmacy Pharmacol 2001, 53:155-166.

43. Gibot L, Lemelle A, Till U, Moukarzel B, Mingotaud AF, Pimienta V, Saint-Aguet P, Rols MP, Gaucher M, Violleau F, Chassenieux C, Vicendo P: Polymeric micelles encapsulating photosensitizer: structure/photodynamic therapy efficiency relation. Biomacromolecules 2014, 15:1443-1455.

44. Koo H, Lee H, Lee S, Min KH, Kim MS, Lee DS, Choi Y, Kwon IC, Kim K, Jeong SY: In vivo tumor diagnosis and photodynamic therapy via tumoral pH-responsive polymeric micelles. Chem Commun (Camb) 2010, 46:5668-5670.

45. van Nostrum CF: Polymeric micelles to deliver photosensitizers for photodynamic therapy. Adv Drug Deliv Rev 2004, 56:9-16.

46. Maeda $H$ : The enhanced permeability and retention (EPR) effect in tumor vasculature: the key role of tumor-selective macromolecular drug targeting. Adv Enzyme Regul 2001, 41:189-207.

47. Nehoff H, Parayath NN, Domanovitch L, Taurin S, Greish K: Nanomedicine for drug targeting: strategies beyond the enhanced permeability and retention effect. Int J Nanomedicine 2014, 9:2539-2555.

48. Li F, Bae BC, Na K: Acetylated hyaluronic acid/photosensitizer conjugate for the preparation of nanogels with controllable phototoxicity: synthesis, characterization, autophotoquenching properties, and in vitro phototoxicity against HeLa cells. Bioconjug Chem 2010, 21:1312-1320.

49. Bae BC, Na K: Self-quenching polysaccharide-based nanogels of pullulan/ folate-photosensitizer conjugates for photodynamic therapy. Biomaterials 2010, 31:6325-6335

50. Li L, Bae BC, Tran TH, Yoon KH, Na K, Huh KM: Self-quenchable biofunctional nanoparticles of heparin-folate-photosensitizer conjugates for photodynamic therapy. Carbohyd Polym 2011, 86:708-715.

51. Oh IH, Min HS, Li L, Tran TH, Lee YK, Kwon IC, Choi K, Kim K, Huh KM: Cancer cell-specific photoactivity of pheophorbide a-glycol chitosan nanoparticles for photodynamic therapy in tumor-bearing mice. Biomaterials 2013, 34:6454-6463.

52. Kim WL, Cho H, Li L, Kang HC, Huh KM: Biarmed poly(ethylene glycol)(pheophorbide a) 2 conjugate as a bioactivatable delivery carrier for photodynamic therapy. Biomacromolecules 2014, 15:2224-2234.
53. Park W, Park SJ, Na K: The controlled photoactivity of nanoparticles derived from ionic interactions between a water soluble polymeric photosensitizer and polysaccharide quencher. Biomaterials 2011, 32:8261-8270

54. Li L, Nurunnabi M, Nafiujjaman M, Lee YK, Huh KM: GSH-mediated photoactivity of pheophorbide a-conjugated heparin/gold nanoparticle for photodynamic therapy. J Control Release 2013, 171:241-250.

55. Li L, Md N, Md N, Jeong YY, Lee YK, Huh KM: A photosensitizer-conjugated magnetic iron oxide/gold hybrid nanoparticle as an activatable platform for photodynamic cancer therapy. J Mater Chem B 2014, 2:2929-2937.

56. Matsumura Y, Maeda $\mathrm{H}$ : A new concept for macromolecular therapeutics in cancer chemotherapy: mechanism of tumoritropic accumulation of proteins and the antitumor agent smancs. Cancer Res 1986, 46:6387-6392.

57. Maeda H, Matsumura $Y$ : Tumoritropic and lymphotropic principles of macromolecular drugs. Crit Rev Ther Drug Carrier Syst 1989, 6:193-210.

58. Ben-Dror S, Bronshtein I, Wiehe A, Roder B, Senge MO, Ehrenberg B: On the correlation between hydrophobicity, liposome binding and cellular uptake of porphyrin sensitizers. Photochem Photobiol 2006, 82:695-701.

59. Love WG, Duk S, Biolo R, Jori G, Taylor PW: Liposome-mediated delivery of photosensitizers: localization of zinc (II)-phthalocyanine within implanted tumors after intravenous administration. Photochem Photobiol 1996, 63:656-661.

60. Casas A, Batlle A: Aminolevulinic acid derivatives and liposome delivery as strategies for improving 5 -aminolevulinic acid-mediated photodynamic therapy. Curr Med Chem 2006, 13:1157-1168.

61. Richter AM, Waterfield E, Jain AK, Canaan AJ, Allison BA, Levy JG: Liposomal delivery of a photosensitizer, benzoporphyrin derivative monoacid ring A (BPD), to tumor tissue in a mouse tumor model. Photochem Photobiol 1993, 57:1000-1006.

62. Namiki $Y$, Namiki T, Date M, Yanagihara $K$, Yashiro M, Takahashi $\mathrm{H}$ : Enhanced photodynamic antitumor effect on gastric cancer by a novel photosensitive stealth liposome. Pharmacol Res Off I Ital Pharmacol Soc 2004, 50:65-76.

63. Sibani SA, McCarron PA, Woolfson AD, Donnelly RF: Photosensitiser delivery for photodynamic therapy. Part 2: systemic carrier platforms. Expert Opin Drug Deliv 2008, 5:1241-1254.

64. Chan JM, Valencia PM, Zhang L, Langer R, Farokhzad OC: Polymeric nanoparticles for drug delivery. Methods Mol Biol 2010, 624:163-175.

65. Veronese FM, Pasut G: PEGylation, successful approach to drug delivery. Drug Discov Today 2005, 10:1451-1458.

66. Jain A, Jain SK: PEGylation: an approach for drug delivery. A review. Crit Rev Ther Drug Carrier Syst 2008, 25:403-447.

67. Kumari A, Yadav SK, Yadav SC: Biodegradable polymeric nanoparticles based drug delivery systems. Colloids Surf B: Biointerfaces 2010, 75:1-18.

68. Allemann E, Brasseur N, Benrezzak O, Rousseau J, Kudrevich SV, Boyle RW, Leroux JC, Gurny R, Van Lier JE: PEG-coated poly(lactic acid) nanoparticles for the delivery of hexadecafluoro zinc phthalocyanine to EMT-6 mouse mammary tumours. J Pharmacy Pharmacol 1995, 47:382-387.

69. Konan YN, Berton M, Gurny R, Allemann E: Enhanced photodynamic activity of meso-tetra(4-hydroxyphenyl)porphyrin by incorporation into sub-200 nm nanoparticles. Eur J Pharm Sci 2003, 18:241-249.

70. Konan YN, Cerny R, Favet J, Berton M, Gurny R, Allemann E: Preparation and characterization of sterile sub-200 nm meso-tetra(4-hydroxylphenyl)porphyrin-loaded nanoparticles for photodynamic therapy. Eur J Pharm Biopharm Off J Arbeitsgemeinschaft Pharm Verfahrenstechnik eV 2003, 55:115-124.

71. Aliabadi HM, Lavasanifar A: Polymeric micelles for drug delivery. Expert Opin Drug Deliv 2006, 3:139-162.

72. Nishiyama N, Kataoka K: Current state, achievements, and future prospects of polymeric micelles as nanocarriers for drug and gene delivery. Pharmacol Ther 2006, 112:630-648.

73. Li B, Moriyama EH, Li F, Jarvi MT, Allen C, Wilson BC: Diblock copolymer micelles deliver hydrophobic protoporphyrin IX for photodynamic therapy. Photochem Photobiol 2007, 83:1505-1512.

74. Shieh MJ, Peng CL, Chiang WL, Wang CH, Hsu CY, Wang SJ, Lai PS: Reduced skin photosensitivity with meta-tetra(hydroxyphenyl)chlorinloaded micelles based on a poly(2-ethyl-2-oxazoline)-b-poly(d, l-lactide) diblock copolymer in vivo. Mol Pharm 2010, 7:1244-1253.

75. Knop K, Mingotaud AF, El-Akra N, Violleau F, Souchard JP: Monomeric pheophorbide(a)-containing poly(ethyleneglycol-b-epsilon-caprolactone) micelles for photodynamic therapy. Photochem Photobiol Sci 2009, 8:396-404. 
76. Li L, Cho H, Yoon KH, Kang HC, Huh KM: Antioxidant-photosensitizer dual-loaded polymeric micelles with controllable production of reactive oxygen species. Int J Pharm 2014, 471:339-348.

77. Muthiah M, Park SH, Nurunnabi M, Lee J, Lee YK, Park H, Lee BI, Min JJ, Park IK: Intracellular delivery and activation of the genetically encoded photosensitizer Killer Red by quantum dots encapsulated in polymeric micelles. Colloid Surface B Biointerfaces 2014, 116:284-294.

78. Bulina ME, Chudakov DM, Britanova OV, Yanushevich YG, Staroverov DB, Chepurnykh TV, Merzlyak EM, Shkrob MA, Lukyanov S, Lukyanov KA: A genetically encoded photosensitizer. Nat Biotechnol 2006, 24:95-99.

79. Liao ZX, Li YC, Lu HM, Sung HW: A genetically-encoded KillerRed protein as an intrinsically generated photosensitizer for photodynamic therapy. Biomaterials 2014, 35:500-508.

80. Pletnev S, Gurskaya NG, Pletneva NV, Lukyanov KA, Chudakov DM, Martynov VI, Popov VO, Kovalchuk MV, Wlodawer A, Dauter Z, Pletnev V: Structural basis for phototoxicity of the genetically encoded photosensitizer KillerRed. J Biol Chem 2009, 284:32028-32039.

81. Ryumina AP, Serebrovskaya EO, Shirmanova MV, Snopova LB, Kuznetsova MM, Turchin IV, Ignatova NI, Klementieva NV, Fradkov AF, Shakhov BE, Zagaynova EV, Lukyanov KA, Lukyanov SA: Flavoprotein miniSOG as a genetically encoded photosensitizer for cancer cells. Biochim Biophys Acta 1830, 2013:5059-5067.

82. Verhille M, Couleaud P, Vanderesse R, Brault D, Barberi-Heyob M, Frochot C: Modulation of photosensitization processes for an improved targeted photodynamic therapy. Curr Med Chem 2010, 17:3925-3943.

83. Lovell JF, Liu TWB, Chen J, Zheng G: Activatable Photosensitizers for Imaging and Therapy. Chem Rev 2010, 110:2839-2857.

84. Bugaj AM: Targeted photodynamic therapy-a promising strategy of tumor treatment. Photochem Photobiol Sci 2011, 10:1097-1109.

85. McCarthy JR, Weissleder R: Model systems for fluorescence and singlet oxygen quenching by metalloporphyrins. ChemMedChem 2007, 2:360-365.

86. Lovell JF, Chen J, Jarvi MT, Cao WG, Allen AD, Liu Y, Tidwell TT, Wilson BC, Zheng G: FRET quenching of photosensitizer singlet oxygen generation. J Phys Chem B 2009, 113:3203-3211.

87. Lee SJ, Koo H, Lee DE, Min S, Lee S, Chen X, Choi Y, Leary JF, Park K, Jeong SY, Kwon IC, Kim K, Choi K: Tumor-homing photosensitizer-conjugated glycol chitosan nanoparticles for synchronous photodynamic imaging and therapy based on cellular on/off system. Biomaterials 2011, 32:4021-4029.

88. Gamcsik MP, Kasibhatla MS, Teeter SD, Colvin OM: Glutathione levels in human tumors. Biomarkers 2012, 17:671-691.

doi:10.1186/2055-7124-18-19

Cite this article as: Li and Huh: Polymeric nanocarrier systems for photodynamic therapy. Biomaterials Research 2014 18:19.

\section{Submit your next manuscript to BioMed Central and take full advantage of:}

- Convenient online submission

- Thorough peer review

- No space constraints or color figure charges

- Immediate publication on acceptance

- Inclusion in PubMed, CAS, Scopus and Google Scholar

- Research which is freely available for redistribution

Submit your manuscript at www.biomedcentral.com/submit
( Biomed Central 